\title{
Nanoparticle-modified magnetic vortex dynamics
}

\author{
Jasper P. Fried, and Peter J. Metaxas, Senior Member, IEEE \\ School of Physics, M013, University of Western Australia, 35 Stirling Hwy, Crawley WA 6009, Australia.
}

\begin{abstract}
With a view towards ferromagnetic-resonancemediated detection of magnetic nanoparticles, we use micromagnetic simulations to study how the stray magnetic field produced by a uniformly magnetized spherical magnetic nanoparticle can modify the vortex gyrotropic resonance. We also show how these modifications depend on the particle's position and size. For small particle sizes, a core confinement effect induced by interaction between the particle's highly localized out-of-plane stray field and the vortex core magnetization can induce large frequency shifts. However, the generation of large shifts via this mechanism relies on the core's trajectory being localized directly below the particle. For larger particles (which generate stronger but less localized stray fields), changes in the gyrotropic frequency result from a combination of wide-scale out-of-plane spin canting and in-plane-field-induced shifting of the vortex core within its anharmonic confining potential. Compared to confinement-driven frequency shifting, the generation of large frequency shifts $(\gtrsim 10$ MHz) via these latter mechanisms is less dependent on particle positioning and the core orbit radius. The use of large particles thus represents a more reliable sensing modality if the particles cannot be guaranteed to align centrally over the disk.
\end{abstract}

Index terms - Nanomagnetics, vortex dynamics, micromagnetic models, magnetic sensors, biomagnetic particles.

\section{INTRODUCTION}

The magnetic vortex configuration arises naturally in (sub)micron sized ferromagnetic disks and consists of an inplane curling magnetization that turns out-of-plane at the vortex's nano-scale core [Fig. 1(a)] [1], [2], [3], [4], [5]. Vortices exhibit a gyrotropic resonance which involves an orbit-like motion of the vortex core within its geometrically induced confining potential [6], [7]. Together with characteristics of the vortex spin structure, the core confining potential determines the frequency of core gyration with more highly confined (stiffer) cores tending to have a higher gyrotropic frequency, $f_{\mathrm{G}}[6]$. Both the vortex spin structure and vortex core confinement can be modified by an external field, $H$ [8], [9], [10], [11], [12], thus enabling field-tuning of $f_{\mathrm{G}}\left(f_{\mathrm{G}}\right.$ can also be modified by geometrical and magnetic inhomogeneities [13], [14], [15], [16]). The field-dependence of $f_{\mathrm{G}}$ has led to the use of vortices for tuneable electronic oscillators [17], [18], data storage [19] and frequency-based [20] magnetic field detection [21], [11].

One potential application of magnetic field detection is in magnetic biosensing for e.g., point-of-care medical diagnostics [22], [23]. In this biosensing approach, functionalized magnetic nanoparticles (MNPs) are used as labels for biological analytes. Exploiting the fact that most biological samples have a negligible magnetic background [24] the presence of these analytes can then be inferred by detecting the stray magnetic field created by the MNP $\operatorname{tag}(\mathrm{s})$. This detection is often

e-mail: peter.metaxas@uwa.edu.au (a)
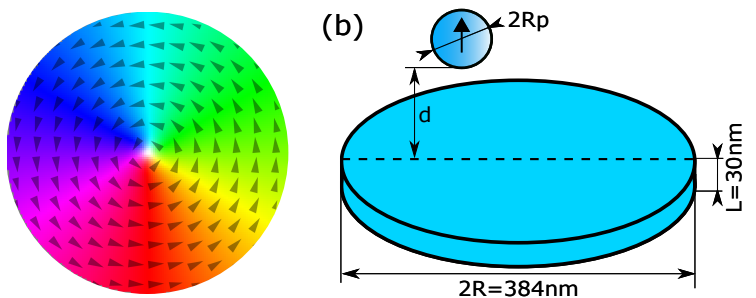

Fig. 1. (a) A simulated magnetic vortex magnetization configuration in a Permalloy-like disk with width $(2 R) 384 \mathrm{~nm}$ and thickness $(L) 30 \mathrm{~nm}$ (zero magnetic field). The arrows show the direction of the in-plane curling magnetization (also encoded via color). The central white spot corresponds to the vortex core. (b) The simulation geometry used in this work: a ferromagnetic disk supporting a vortex together with an out-of-plane magnetized MNP (radius $=R_{P}$ ) which is modeled as a dipole magnetic field source located at a height, $d+R_{P}$, above the top surface of the disk.

achieved using Hall Effect sensors or conventional tunneling or giant magnetoresistive field sensors [23] (in the latter, MNP fields modify the quasi-static magnetization in the device's sensing layer, leading to a change in the device resistance [25]). MNP fields can however also modify vortex dynamics (and statics) [9], [11] and these dynamics can be probed electrically [17], [18], [26], opening pathways for vortexbased, electronic biosensors [21], [11]. Magnetic biosensing techniques that are based on (electrically) detecting changes in the frequency of resonant magnetization dynamics [20], [27], [28], [29] contrast the more conventional approaches outlined above in that they can operate naturally in the frequency domain (typically $\sim 0.1-10 \mathrm{GHz}$ ). Other potential advantages of a frequency based approach include fast sensor response times (e.g., [30], [31]), highly promising size scalability [20] and a wide operational external field range [32], the latter enabling the generation of large particle moments [28].

In this paper we use micromagnetic simulations to study how the stray magnetic field produced by a uniformly magnetized, spherical MNP can modify the vortex gyrotropic resonance frequency. Specifically, we show how the observation of significant MNP-induced frequency shifts $(\sim 10 \mathrm{MHz}$ or more) as well as the mechanisms underlying these shifts depends on particle size, particle position and core orbit radius.

\section{Micromagnetic Simulation MEthod}

Simulations were run using MuMax3 [33] with the system geometry shown in Fig. 1(b). The disk (diameter $2 R=384 \mathrm{~nm}$ and thickness $L=30 \mathrm{~nm}$ ) is Permalloy-like: saturation magnetization $M_{\mathrm{S}}=800 \mathrm{kA} \mathrm{m}^{-1}$; exchange constant $A_{e x}=13$ $\mathrm{pJ} \mathrm{m}^{-1}$; nil intrinsic magnetic anisotropy; damping constant $\alpha=0.008$; gyromagnetic ratio $\gamma=1.7595 \times 10^{11} \operatorname{rad}(\mathrm{T} . \mathrm{s})^{-1}$ (within $\sim 5 \%$ of the experimentally determined value [34]). A rectangular discretization with a cell size of $3 \times 3 \times 3.75$ 
$\mathrm{nm}^{3}$ was employed. We will consider the case of a vortex with an anticlockwise chirality and a polarity aligned with the particle's magnetization $(p=+1)$. Note the polarity can be set experimentally with an out-of-plane (OOP) magnetic field (e.g., [8]). The polarity also defines the sign of the change in $f_{\mathrm{G}}$ due to OOP fields [8].

The MNPs, MNP clusters or MNP-embedded polymer beads used in biosensing are typically superparamagnetic [23]. As such, an external field is required to obtain a finite timeaveraged MNP magnetic moment which will be maximized in large fields. The OOP field geometry is preferable for vortexbased sensing in terms of maximizing the MNP moment without destroying the vortex state: the in-plane annihilation field is typically 10's of mT [1] whereas the out-of-plane saturation field is 100's of mT [8]. We will treat the MNP as a $+z$ magnetized magnetic dipole located at a height of $d+R_{\mathrm{P}}$ above the disk (as in [11]). This allows us to limit the size of the simulation geometry to a cuboid containing only the disk. Physically, $d(10-100 \mathrm{~nm})$ represents the separation between the bottom of the spherical particle and the top of the disk (passivation layers and contacts will limit the minimum $d$ in real devices [24]). For simplicity, most simulations are carried out in zero external magnetic field and we artificially set $M_{\mathrm{S}, \mathrm{P}}$ (the MNP's $M_{\mathrm{S}}$ ) to $200 \mathrm{kA} / \mathrm{m}$ (the dipole moment is calculated as $\frac{4}{3} \pi R_{\mathrm{P}}^{3} M_{\mathrm{S}, \mathrm{P}}$ ). Note that a (relatively easy to generate) $200 \mathrm{mT}$ field can induce this moment in magnetically clustered MNPs [27] and this (field-dependent, non-saturated) moment has been successfully used in simulations to reproduce experimentally observed MNP-modified resonances [28]. We will however confirm that comparbale results are obtained in a finite OOP field.

For each simulation, we set a vortex-like magnetic state, optionally add a dipole and/or external magnetic field and then compute the equilibrium magnetization configuration in the ferromagnetic disk using MuMax3's internal relaxation routine, 'relax()'. We concentrate first on results where the gyrotropic mode is excited through the application of a transverse field pulse with a sinc function time dependence (frequency cutoff of $30 \mathrm{GHz}$, offset of $300 \mathrm{ps}$ and amplitude of $2 \mathrm{mT}$ ). Such a field induces a displacement of the vortex core which is followed by damped, small amplitude $(\sim 1 \mathrm{~nm})$ gyrotropic core dynamics (referred to here as 'ringdown' dynamics). We extract $f_{\mathrm{G}}$ from a discrete Fourier transform of the time dependent, $x$-component of the spatially averaged disk magnetization (total time $=100 \mathrm{~ns}$ ). In the absence of an applied field, $f_{\mathrm{G}}=f_{\mathrm{G}, 0} \approx 580 \mathrm{MHz}$. We will mostly consider changes in $f_{\mathrm{G}}$ measured relative to $f_{\mathrm{G}, 0}\left(\Delta f_{\mathrm{G}}=f_{\mathrm{G}}-f_{\mathrm{G}, 0}\right)$.

\section{PARTICLE-DRIVEN GYRotropic FREQUenCy SHIFTING}

There are three mechanisms by which a MNP's stray field can modify $f_{\mathrm{G}}$. Below, we consider how these mechanisms change the core stiffness coefficient, $\kappa$, and/or the gyroconstant, $G$, which together determine $f_{\mathrm{G}}$ via [6]:

$$
2 \pi f_{\mathrm{G}}=\frac{\kappa}{G} \text {. }
$$
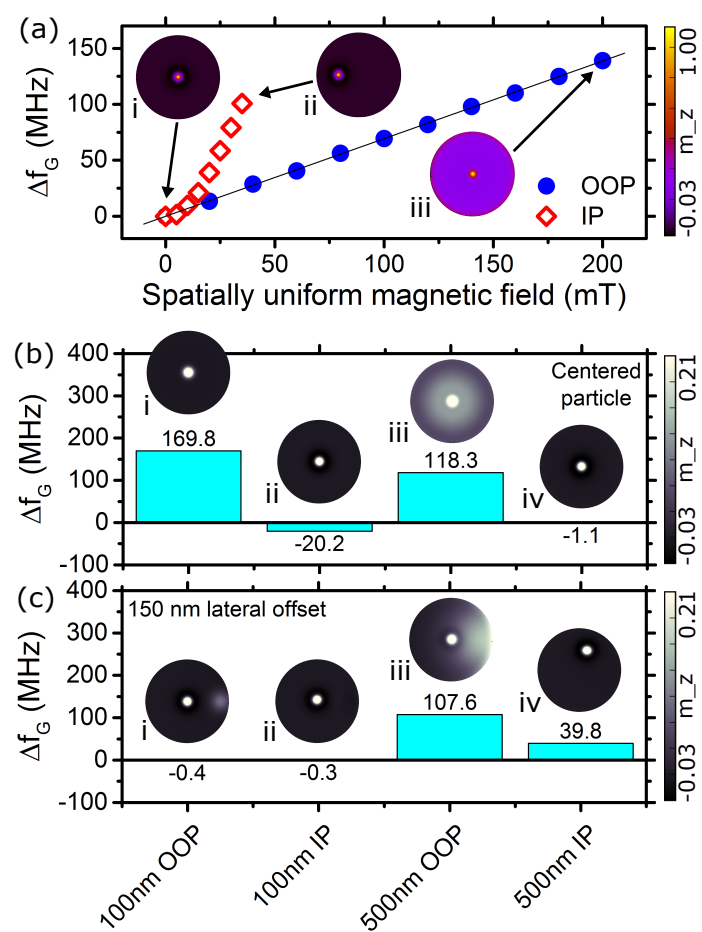

Fig. 2. (a) $\Delta f_{\mathrm{G}}$ as a function of in-plane (IP) or out-of-plane (OOP) magnetic field (static and spatially uniform). The thin black line is a linear fit to the OOP data going through $(0,0)$. Insets (i-iii) show maps of the $z$-component of the equilibrium magnetization configuration $\left(m_{z}\right)$ for the fields marked by the arrows $\left(m_{z}-\right.$ range is -0.03 to 1$)$. One can identify the core position (light dot) and/or disk-wide spin canting [disk-wide purple shading, e.g., inset (iii)]. $(\mathrm{b}, \mathrm{c}) \Delta f_{\mathrm{G}}$ values induced by IP or OOP field components of a particle with width, $2 R_{P}$, of $100 \mathrm{~nm}$ or $500 \mathrm{~nm}$ with a disk-particle separation, $d$, of 10 $\mathrm{nm}$. Data is shown for a particle which is (b) laterally centered or (c) offset by $150 \mathrm{~nm}$ in the $+x$ direction. As above, at-equilibrium maps of $m_{z}$ are shown for each condition: insets (i-iv). The $m_{z}$-range here is -0.03 to 0.21 . This facilitates visualization of localized canting but makes the vortex core $\left(m_{z}=1\right.$ at its center) appear wider.

$\kappa$ describes the restoring force acting on the vortex core when the core is displaced from its equilibrium position. For small radial core displacements, $X$, one can assume a parabolic scaling of the disk's magnetic energy, $W: W(X)=$ $W(0)+\frac{1}{2} \kappa X^{2}$ (the confinement potential is however typically anharmonic in reality [9]). The main contribution to $\kappa$ is typically magnetostatic [6], [35] however exchange or Zeeman energies [12], [11] and Oersted fields [36] can be important in certain scenarios. The gyroconstant, $G$, describes the strength of the lateral force acting on the vortex core due to its motion. It can be calculated from the thickness averaged spin structure using $G=\left(M_{\mathrm{S}} L / \gamma\right) \int_{A} \mathbf{m} \cdot(d \mathbf{m} / d x \times d \mathbf{m} / d y) d x d y$ [37], [38], [6] where $\mathbf{m}$ is the normalized magnetization vector. For small amplitude motion it has been shown that the integration should be carried out over the vortex core [11].

The three mechanisms by which a dipole field can modify $f_{\mathrm{G}}$ (Eq. (1)) are as follows. (i) 'Core confinement' occurs when localized out-of-plane fields aligned with the core magnetization are centered above the vortex core [11]. These fields confine the core, making lateral displacements energetically unfavorable. This increases $\kappa$ and thus $f_{\mathrm{G}}$. (ii) 'Spin canting' arises in the presence of spatially (quasi)uniform OOP fields which generate an OOP canting of the vortex's in-plane curling 
magnetization [Fig. 2(a), inset iii]. Canting parallel to the core reduces both $\kappa$ and $G$ [8], [12]. However, reductions in $G$ dominate, resulting in an increased $f_{\mathrm{G}}$ [8], [12]. (iii) 'Core shifting' occurs in the presence of IP fields which shift the vortex core within its anharmonic confining potential [9]. In symmetric disks, shifts of the core away from the disk's center increase $\kappa$ and thus $f_{\mathrm{G}}$ [10], [9] [Fig. 2(a), inset ii].

In Fig. 2(b) we show the $\Delta f_{\mathrm{G}}$ induced independently by the IP and OOP components of dipole fields generated by particles with $100 \mathrm{~nm}$ or $500 \mathrm{~nm}$ diameters centered above the disk with $d=10 \mathrm{~nm}$. The OOP field component results in a positive frequency shift for both particle sizes [Figs. 2(b)(i,iii)]. Note that $\Delta f_{\mathrm{G}}$ is larger for the $100 \mathrm{~nm}$ particle despite its smaller volume leading to a weaker stray field. This is because the $100 \mathrm{~nm}$ particle's highly localized field leads to significant core confinement which strongly enhances the $\Delta f_{\mathrm{G}}$ per unit field when compared to the larger particle's wide-scale spin canting [11]. Note that due to the laterally centered position of the particle, the IP components of the centered MNPs' fields are oriented radially inward toward the core and primarily modify the curling magnetization via an inward canting of the spins. For the $100 \mathrm{~nm}$ MNP, this generates a weak, negative $\Delta f_{\mathrm{G}}$ [Fig. 2(b)(ii)].

For a lateral, rightward $(+x)$ shift of the MNP position of $150 \mathrm{~nm}$, only the $500 \mathrm{~nm}$ MNP induces a clear $\Delta f_{\mathrm{G}}$ [Fig. 2(c)]. Although the $100 \mathrm{~nm}$ MNP generates changes in the curling magnetization, these are localized far from the core position [e.g., Fig. 2(c)(i)] and lead to $\Delta f_{\mathrm{G}} \sim 0$. In contrast, the $500 \mathrm{~nm}$ MNP creates a broad stray field distribution, its OOP component generating an OOP spin-canting that is concentrated beneath the particle on the disk's right hand side. Visible canting does however extend to the core's location [unlike for the smaller MNP in Fig. 2(c)(i)], leading to an increase in $f_{\mathrm{G}}$ [Fig. 2(c)(iii)]. We also note that due to the MNP's offset position, the IP component of this MNP's dipolar field is no longer radially oriented towards the disk's center and thus can act on the vortex's curling spin structure to strongly shift the core's position [Fig. 2(c)(iv)], again increasing $f_{\mathrm{G}}$.

In Fig. 3(a) we present data for four different particle sizes ( $d=10 \mathrm{~nm}$ ) showing how $\Delta f_{\mathrm{G}}$ depends on the lateral dipole position along a line passing through the center of the disk for full '3-dimensional' dipole fields (i.e. IP and OOP components). Also shown is the particle's out-of-plane (solid black lines) and in-plane (dashed black lines) fields as calculated at the lateral center of the disk (averaged across the disk's thickness) and the equilibrium core displacement for each dipole position [Fig. 3(b)]. As seen above, the smallest particles $(100 \mathrm{~nm}$ and $200 \mathrm{~nm}$ widths) generate shifts only when close to the center of the disk with $\Delta f_{\mathrm{G}} \approx 0$ for displacements above $\sim R_{\mathrm{P}}$. For centered dipoles, $\Delta f_{\mathrm{G}}$ is about equal for the two smallest MNPs despite the $100 \mathrm{~nm}$ particle generating a much weaker (but more localized) field, a consequence of core confinement. For the two larger particle sizes, $\Delta f_{\mathrm{G}}$ is greater than $10 \mathrm{MHz}$ even at the maximum dipole offset of $300 \mathrm{~nm}$ with two peaks appearing in the $\Delta f_{\mathrm{G}}$ versus position curve. Indeed, the comparatively stronger and less localized IP field component of these larger MNPs remains capable of strongly shifting the core equilibrium position (and thus
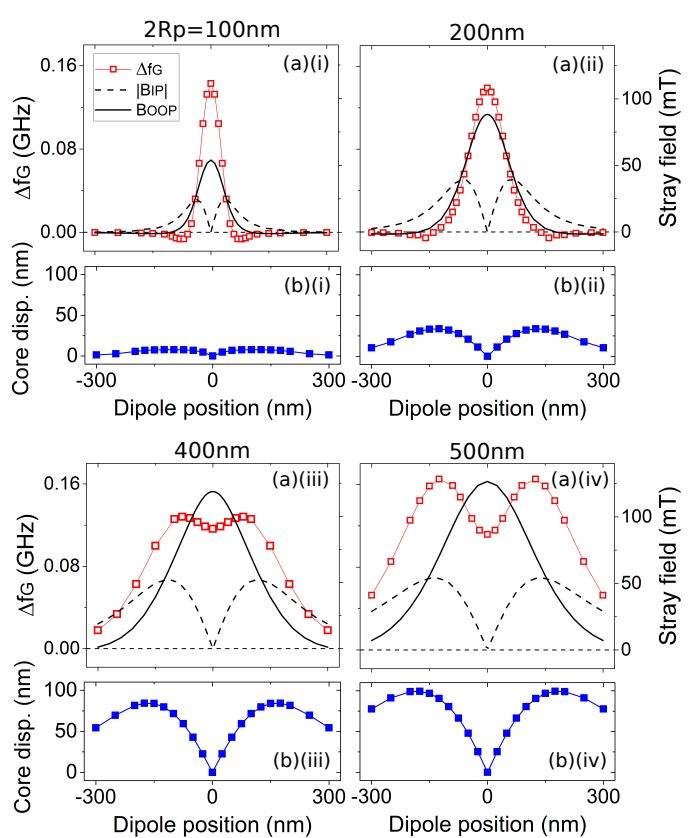

Fig. 3. (a) $\Delta f_{\mathrm{G}}$ (red squares) due to the full particle-generated stray field (i.e. both IP and OOP field components) as a function of particle position for a (i) $100 \mathrm{~nm}$, (ii) $200 \mathrm{~nm}$, (iii) $400 \mathrm{~nm}$ and (iv) $500 \mathrm{~nm}$ MNP. Also shown in these figures is the particle's out-of-plane (solid black lines) and in-plane (dashed black lines) field as calculated at the disk's center. (b) The distance of the vortex core from the disk center as a function of particle position for each particle size.

modifying $f_{\mathrm{G}}$ ) even for large dipole displacements [10's of nm in Figs. 3(a,b)(iii,iv)]. We find that the maximum $\Delta f_{\mathrm{G}}$ arises for dipole positions where the IP field component at the center of the disk is close to maximized. Note that for the large particles that are centered above the disk (i.e. where there is no lateral core displacement and confinement effects are weak due to the large MNPs' broad stray fields) there is still a significant $\Delta f_{\mathrm{G}}$ due to spin-canting [Fig. 2(b)(iii)].

An increased disk-particle separation reduces $\Delta f_{\mathrm{G}}$ [Fig. 4] since the dipolar field acting on the disk is weakened. The percentage reduction in $\Delta f_{\mathrm{G}}$ per unit of vertical displacement is however clearly strongest for the $100 \mathrm{~nm}$ particle since the weaker and broader stray field profile acting on the disk
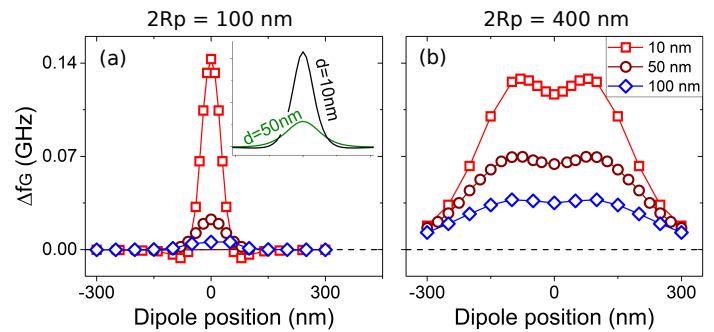

Fig. 4. (a,b) $\Delta f_{\mathrm{G}}$ due to the full particle-generated stray field (i.e. both IP and OOP field components) as a function of particle position for three diskparticle separations for (a) $100 \mathrm{~nm}$ and (b) $400 \mathrm{~nm}$ particles. The inset to (a) plots the out-of-plane stray field at the disk center (averaged across the disk thickness) as a $100 \mathrm{~nm}$ particle is shifted laterally across the disk at a height of $d=10 \mathrm{~nm}$ and $d=50 \mathrm{~nm}$ ( $y$-axis ranges from $-5 \mathrm{mT}$ to $65 \mathrm{mT}, x$-axis ranges from $-200 \mathrm{~nm}$ to $200 \mathrm{~nm}$ ). 
for increased $d$ [Fig. 4(a) inset] significantly reduces coreconfinement [11]. We do however observe a qualitatively similar particle-position- and particle-size-dependence of $\Delta f_{\mathrm{G}}$ : a single peak for the $100 \mathrm{~nm}$ MNP and a double peak for the $400 \mathrm{~nm}$ MNP. Note however that for the $400 \mathrm{~nm}$ particle, the shoulders in the $\Delta f_{\mathrm{G}}$ profile (linked to core shifting) become less distinct as $d$ is increased, consistent with a decreased core displacement due to a weaker stray field. We finally note that the simulated $\Delta f_{\mathrm{G}}$ values for the $400 \mathrm{~nm}$ particle well exceed typically measured vortex nano-oscillator linewidths (down to $\sim 1 \mathrm{MHz}$ or less) [17], [18], [39], [40] which is encouraging for electronic detection (this is also true for the smaller particles, but with stricter restrictions on the particle position).

\section{LARGE AMPLITUDE CORE DYNAMICS}

Instead of small amplitude ringdown dynamics, we now consider larger (and more experimentally relevant; e.g., [41]) core orbits. Here we apply a transverse, radiofrequency magnetic field with amplitude $0.8 \mathrm{mT}$ and frequency close to the $f_{\mathrm{G}}$ determined from the ringdown simulations. This induces gyrotropic core motion (steady state orbit radius $\approx 50 \mathrm{~nm}$ with gyration around the core's equilibrium position) which we refer to as 'driven' dynamics. The driving frequency was stepped and that which induced the largest orbit radius after $50 \mathrm{~ns}$ was taken to be $f_{\mathrm{G}}$ (a $5 \mathrm{MHz}$ step size yielded an intrinsic uncertainty in the extracted $f_{\mathrm{G}}$ of $\pm 2.5 \mathrm{MHz}$ ).

$\Delta f_{\mathrm{G}}$ values as a function of particle position for ringdown (red squares) and driven (blue diamonds) gyrotropic dynamics are compared in Figs. 5(a)-(c). When the particle is placed at the disk center, there is a clear reduction in $\Delta f_{\mathrm{G}}$ for large amplitude, driven oscillations of the vortex core. This reduction is most significant for small particle sizes [simulated down to $2 R_{\mathrm{P}}=40 \mathrm{~nm}$; Fig. 5(a)] with $\Delta f_{\mathrm{G}}$ falling by $\sim 88 \%$ for the $40 \mathrm{~nm}$ MNP compared to $\sim 23 \%$ for the $400 \mathrm{~nm}$ MNP. The strong reduction in $\Delta f_{\mathrm{G}}$ for small MNPs and driven dynamics is consistent with the vortex core trajectory now being outside of the strongest part of the MNP's localized out-of-plane stray field. This weakens localized-field-induced confinement effects, decreasing $\Delta f_{\mathrm{G}}$. As the MNP size is increased however, the reduction in $\Delta f_{\mathrm{G}}$ becomes less significant since the $\Delta f_{\mathrm{G}}$ for large particles is due to wide-scale spin-canting across the disk which will be present throughout the core orbit. Changes in the gyrotropic frequency due to MNP-induced shifts in the core's equilibrium position are also still present for driven dynamics. As such, the characteristic double peak in $\Delta f_{\mathrm{G}}$ for large MNPs is observed for both ringdown and driven dynamics [Fig. 5(c)].

Finally, we show $\Delta f_{\mathrm{G}}$ (determined from ringdown dynamics) as a function of particle position for a $100 \mathrm{~nm}$ and 400 $\mathrm{nm}$ MNP in the presence of a spatially uniform $200 \mathrm{mT}$ outof-plane field [Fig. 5(d)]. We observe a similar particle size dependence of $\Delta f_{\mathrm{G}}$ [now measured with respect to $f_{\mathrm{G}}$ in a 200 mT OOP field; see Fig. 2(a)]. Our results appear to be qualitatively independent of the presence of an out-of-plane magnetic field as long as that field is not so large as to destroy the vortex state or lead to a deviation in the linear OOP field dependence of $f_{\mathrm{G}}[12]$.
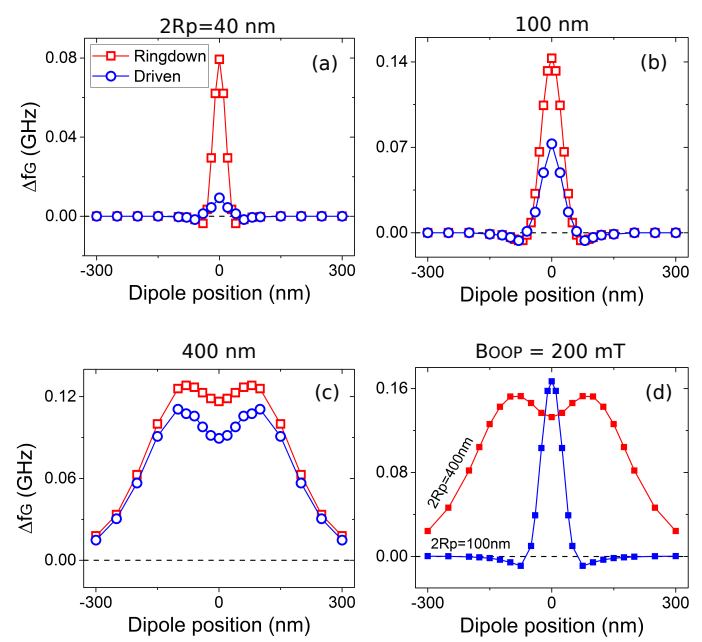

Fig. 5. (a-c) $\Delta f_{\mathrm{G}}$ due to the full particle-generated stray field (i.e. both IP and OOP field components) as a function of particle position for sinc-fielddriven ringdown dynamics (red squares) and sinusoidal-field-driven dynamics (blue diamonds) for a (a) $40 \mathrm{~nm}$ (b) $100 \mathrm{~nm}$ and (c) $400 \mathrm{~nm}$ particle. The diskparticle separation is $d=10 \mathrm{~nm}$. (d) $\Delta f_{\mathrm{G}}$ as a function of particle position in the presence of a spatially uniform $200 \mathrm{mT}$ out-of-plane field (we neglect any influence of stray fields generated by the disk's canted magnetization on the particle moment). This is shown for a $100 \mathrm{~nm}$ diameter particle (blue squares) and a $400 \mathrm{~nm}$ diameter particle (red circles).

\section{CONCLUSION}

In summary, both the IP and OOP components of MNPgenerated stray fields can significantly modify the gyrotropic vortex resonance frequency, $f_{\mathrm{G}}$, in a ferromagnetic disk $\left(\Delta f_{\mathrm{G}} \gtrsim 10\right.$ 's of $\left.\mathrm{MHz}\right)$. Although small particles $(\leq 200 \mathrm{~nm}$ in width in this study) can produce significant changes in $f_{\mathrm{G}}$ via core confinement, these changes are strongly reduced when the core trajectory is not localized directly beneath the particle. In contrast, larger particles $(\geq 400 \mathrm{~nm})$ can create strong changes in $f_{\mathrm{G}}$ even when they are $300 \mathrm{~nm}$ from the vortex core. This is because these particles create broad, strong stray field profiles which modify the gyrotropic frequency via widescale canting of the vortex's curling spins and significant shifts of the core within its confining potential. The observation of a large $\Delta f_{\mathrm{G}}$ due to these latter frequency shifting mechanisms is less dependent on particle placement and core orbit size. As such, the use of larger particles likely represents a more reliable sensing approach, especially if particle position cannot be precisely controlled.

\section{ACKNOWLEDGMENT}

This work was supported by a research grant from the United States Air Force (Asian Office of Aerospace Research and Development, AOARD), the Australian Research Council's Discovery Early Career Researcher Award scheme (DE120100155), the University of Western Australia (Early Career Researcher Fellowship Support and Research Collaboration Award schemes) and by resources provided by the Pawsey Supercomputing Centre with funding from the Australian Government and the Government of Western Australia. 


\section{REFERENCES}

[1] R. P. Cowburn, D. K. Koltsov, A. O. Adeyeye, and M. E. Welland, "Single-domain circular nanomagnets," Phys. Rev. Lett., vol. 83, p. 1042, 1999.

[2] T. Shinjo, T. Okuno, R. Hassdorf, K. Shigeto, and T. Ono, "Magnetic vortex core observation in circular dots of permalloy," Science, vol. 289, no. 5481, p. 930, 2000

[3] A. Wachowiak, J. Wiebe, M. Bode, O. Pietzsch, M. Morgenstern, and R. Wiesendanger, "Direct observation of internal spin structure of magnetic vortex cores," Science, vol. 298, no. 5593, pp. 577-580, Oct 2002.

[4] J. K. Ha, R. Hertel, and J. Kirschner, "Micromagnetic study of magnetic configurations in submicron permalloy disks," Phys. Rev. B, vol. 67, p. 224432, Jun 2003.

[5] K. Y. Guslienko and V. Novosad, "Vortex state stability in soft magnetic cylindrical nanodots," J. Appl. Phys, vol. 96, p. 4451, 2004.

[6] K. Y. Guslienko, B. A. Ivanov, V. Novosad, Y. Otani, H. Shima, and K. Fukamichi, "Eigenfrequencies of vortex state excitations in magnetic submicron-size disks," J. Appl. Phys., vol. 91, no. 10, pp. 8037-8039, 2002.

[7] V. Novosad, F. Y. Fradin, P. E. Roy, K. S. Buchanan, K. Y. Guslienko, and S. D. Bader, "Magnetic vortex resonance in patterned ferromagnetic dots," Phys. Rev. B, vol. 72, p. 024455, 2005.

[8] G. de Loubens, A. Riegler, B. Pigeau, F. Lochner, F. Boust, K. Y Guslienko, H. Hurdequint, L. W. Molenkamp, G. Schmidt, A. N. Slavin, V. S. Tiberkevich, N. Vukadinovic, and O. Klein, "Bistability of vortex core dynamics in a single perpendicularly magnetized nanodisk," Phys. Rev. Lett., vol. 102, no. 17, p. 177602, May 2009.

[9] O. V. Sukhostavets, B. Pigeau, S. Sangiao, G. de Loubens, V. V. Naletov, O. Klein, K. Mitsuzuka, S. Andrieu, F. Montaigne, and K. Y. Guslienko, "Probing the anharmonicity of the potential well for a magnetic vortex core in a nanodot," Phys. Rev. Lett., vol. 111, no. 24, p. 247601, Dec 2013.

[10] K. S. Buchanan, P. E. Roy, M. Grimsditch, F. Y. Fradin, K. Y. Guslienko, S. D. Bader, and V. Novosad, "Magnetic-field tunability of the vortex translational mode in micron-sized permalloy ellipses: Experiment and micromagnetic modeling," Phys. Rev. B, vol. 74, p. 064404, 2006.

[11] J. P. Fried and P. J. Metaxas, "Localized magnetic fields enhance the field sensitivity of the gyrotropic resonance frequency of a magnetic vortex," Phys. Rev. B, vol. 93, p. 064422, Feb 2016.

[12] J. P. Fried, H. Fangohr, M. Kostylev, and P. J. Metaxas, "Exchangemediated, non-linear, out-of-plane magnetic field dependence of the ferromagnetic vortex gyrotropic mode frequency driven by core deformation," Phys. Rev. B, vol. 94, p. 224407, 2016

[13] R. L. Compton and P. A. Crowell, "Dynamics of a pinned magnetic vortex," Phys. Rev. Lett., vol. 97, p. 137202, Sep 2006.

[14] R. L. Compton, T. Y. Chen, and P. A. Crowell, "Magnetic vortex dynamics in the presence of pinning," Phys. Rev. B, vol. 81, no. 14, p. 144412, Apr 2010.

[15] J. H. Silva, D. Toscano, F. Sato, P. Z. Coura, B. V. Costa, and S. A. Leonel, "The influence of magnetic impurities in the vortex core dynamics in magnetic nano-disks," J. Magn. Magn. Mat., vol. 324 no. 19 , p. 3083,2012

[16] D. Toscano, S. A. Leonel, P. Z. Coura, F. Sato, R. A. Dias, and B. V. Costa, "Dynamics of the vortex core in magnetic nanodisks with a ring of magnetic impurities," Appl. Phys. Lett., vol. 101, no. 25, p. 252402, 2012.

[17] V. S. Pribiag, I. N. Krivorotov, G. D. Fuchs, P. M. Braganca, O. Ozatay, J. C. Sankey, D. C. Ralph, and R. A. Buhrman, "Magnetic vortex oscillator driven by d.c. spin-polarized current," Nat. Phys., vol. 3, p. 498, 2007.

[18] A. Dussaux, B. Georges, J. Grollier, V. Cros, A. V. Khvalkovskiy, A. Fukushima, M. Konoto, H. Kubota, K. Yakushiji, S. Yuasa, K. A Zvezdin, K. Ando, and A. Fert, "Large microwave generation from current-driven magnetic vortex oscillators in magnetic tunnel junctions," Nat. Commun., vol. 1, p. 8, 2010.

[19] B. Pigeau, G. de Loubens, O. Klein, A. Riegler, F. Lochner, G. Schmidt, L. W. Molenkamp, V. S. Tiberkevich, and A. N. Slavin, "A frequencycontrolled magnetic vortex memory," Appl. Phys. Lett., vol. 96, no. 13, p. $132506,2010$.

[20] P. M. Braganca, B. A. Gurney, B. A. Wilson, J. A. Katine, S. Maat, and J. R. Childress, "Nanoscale magnetic field detection using a spin torque oscillator," Nanotechnol., vol. 21, no. 23, p. 235202, 2010.

[21] P. Wohlhüter, M. T. Bryan, P. Warnicke, S. Gliga, S. E. Stevenson, G. Heldt, L. Saharan, A. K. Suszka, C. Moutafis, R. V. Chopdekar, J. Raabe, T. Thomson, G. Hrkac, and L. J. Heyderman, "Nanoscale switch for vortex polarization mediated by bloch core formation in magnetic hybrid systems," Nat. Comms., vol. 6, p. 7836, Aug 2015.

[22] P. P. Freitas, F. A. Cardoso, V. C. Martins, S. A. M. Martins, J. Loureiro, J. Amaral, R. C. Chaves, S. Cardoso, L. P. Fonseca, A. M. Sebastião, and et al., "Spintronic platforms for biomedical applications," Lab Chip, vol. 12 , no. 3, pp. 546-557, 2012.

[23] H. Lee, T.-H. Shin, J. Cheon, and R. Weissleder, "Recent developments in magnetic diagnostic systems," Chem. Rev., vol. 115, no. 19, p. 10690, Oct 2015.

[24] R. S. Gaster, D. A. Hall, C. H. Nielsen, S. J. Osterfeld, H. Yu, K. E. Mach, R. J. Wilson, B. Murmann, J. C. Liao, S. S. Gambhir et al., "Matrix-insensitive protein assays push the limits of biosensors in medicine," Nat. Med., vol. 15, p. 1327, 2009.

[25] J.-R. Lee, N. Sato, D. J. B. Bechstein, S. J. Osterfeld, J. Wang, A. W. Gani, D. A. Hall, and S. X. Wang, "Experimental and theoretical investigation of the precise transduction mechanism in giant magnetoresistive biosensors," Sci. Rep., vol. 6, p. 18692, Jan 2016.

[26] J.-V. Kim, "Spin-torque oscillators," Solid State Phys., vol. 63, pp. 217 - 294, 2012.

[27] P. J. Metaxas, M. Sushruth, R. Begley, J. Ding, R. C. Woodward, I. Maksymov, M. Albert, W. Wang, H. Fangohr, A. Adeyeye, and M. Kostylev, "Sensing magnetic nanoparticles using nano-confined ferromagnetic resonances in a magnonic crystal," Appl. Phys. Lett., vol. 106, p. 232406, Jan. 2015.

[28] M. Sushruth, J. Ding, J. Duczynski, R. C. Woodward, R. Begley, H. Fangohr, R. O. Fuller, A. O. Adeyeye, M. Kostylev, and P. J. Metaxas, "Resonance-based detection of magnetic nanoparticles and microbeads using nanopatterned ferromagnets," Phys. Rev. Appl., vol. 6, p. 044005, 2016.

[29] M. Albert, M. Beg, D. Chernyshenko, M.-A. Bisotti, R. L. Carey, H. Fangohr, and P. J. Metaxas, "Frequency-based nanoparticle sensing over large field ranges using the ferromagnetic resonances of a magnetic nanodisc," Nanotechnol., vol. 27, p. 455502, 2016.

[30] K. Mizushima, K. Kudo, T. Nagasawa, and R. Sato, "Signal-to-noise ratios in high-signal-transfer-rate read heads composed of spin-torque oscillators," J. Appl. Phys., vol. 107, no. 6, p. 063904, 2010.

[31] T. Nagasawa, H. Suto, K. Kudo, K. Mizushima, and R. Sato, "Response of magnetic tunnel junction-based spin-torque oscillator to series of subnanosecond magnetic pulses," Appl. Phys Express, vol. 9, no. 11, p. 113002 , oct 2016

[32] J. R. Petrie, S. Urazhdin, K. A. Wieland, G. A. Fischer, and A. S Edelstein, "Using a spin torque nano-oscillator to read memory based on the magnetic permeability," J. Phys. D: Appl. Phys., vol. 47, no. 5, p. 055002, Jan 2014.

[33] A. Vansteenkiste, J. Leliaert, M. Dvornik, M. Helsen, F. Garcia-Sanchez, and B. Van Waeyenberg, "The design and verification of MuMax3," AIP Adv., vol. 4, p. 107133, 2014.

[34] J. M. Shaw, H. T. Nembach, T. J. Silva, and C. T. Boone, "Precise determination of the spectroscopic g-factor by use of broadband ferromagnetic resonance spectroscopy," J. Appl. Phys, vol. 114, no. 24, p. 243906, Dec 2013.

[35] K. Y. Guslienko, X. F. Han, D. J. Keavney, R. Divan, and S. D. Bader, "Magnetic vortex core dynamics in cylindrical ferromagnetic dots," Phys. Rev. Lett., vol. 96, no. 6, p. 067205, Feb 2006.

[36] A. Dussaux, A. V. Khvalkovskiy, P. Bortolotti, J. Grollier, V. Cros, and A. Fert, "Field dependence of spin-transfer-induced vortex dynamics in the nonlinear regime," Phys. Rev. B, vol. 86, no. 1, Jul 2012.

[37] A. Thiele, "Steady-state motion of magnetic domains," Phys. Rev. Lett., vol. 30, no. 6, p. 230, Feb 1973.

[38] D. L. Huber, "Dynamics of spin vortices in two-dimensional planar magnets," Phys. Rev. B, vol. 26, no. 7, p. 3758, Oct 1982.

[39] N. Locatelli, V. V. Naletov, J. Grollier, G. de Loubens, V. Cros, C. Deranlot, C. Ulysse, G. Faini, O. Klein, and A. Fert, "Dynamics of two coupled vortices in a spin valve nanopillar excited by spin transfer torque," Appl. Phys. Lett., vol. 98, p. 062501, 2011.

[40] R. Lebrun, N. Locatelli, S. Tsunegi, J. Grollier, V. Cros, F. Abreu Araujo, H. Kubota, K. Yakushiji, A. Fukushima, and S. Yuasa, "Nonlinear behavior and mode coupling in spin-transfer nano-oscillators," Phys. Rev. App., vol. 2, no. 6, p. 061001, 2014.

[41] A. S. Jenkins, R. Lebrun, E. Grimaldi, S. Tsunegi, P. Bortolotti, H. Kubota, K. Yakushiji, A. Fukushima, G. de Loubens, O. Klein, S. Yuasa, and V. Cros, "Spin torque resonant vortex core expulsion for an efficient radio-frequency detection scheme," Nat. Nanotechnol., vol. 11, p. 360, May 2016. 